\title{
Basic urinal flow curves classification with proposed solutions
}

\author{
Dominik Stursa ${ }^{1}$, Petr Dolezel ${ }^{1}$, and Daniel Honc ${ }^{1}$ \\ University of Pardubice, Pardubice, Czech Republic, \\ dominik.stursa@upce.cz, \\ WWW home page: http://www.upce.cz/fei
}

\begin{abstract}
Nowadays, the pressure on prevent invasive methods for diagnostics is still increasing in the health care sector. In the case of the lower urinary tract, early diagnosis can play a significant role to prevent a surgery. Here, the widely used non-invasive test, the uroflowmetry, is observed. As the new measurement devices are being created, new algorithms for basic urinary flow classification must be developed. There, the feature extraction methods are developed and introduced for further use in combination with standard classifiers based on machine learning. In the further work, the methods will be reviewed on extensive dataset, which is currently being created. As the credible dataset verified by several urologist will be obtained, the proposed methods should be examined. Direction of further development will depend on the results of introduced methods.
\end{abstract}

Keywords: Uroflowmetry, urine flow classification, feature extraction, pattern recognition.

\section{Introduction}

Nowadays, the pressure on prevent invasive methods for diagnostics is still increasing in the health care sector. In the case of the lower urinary tract, early diagnosis can play a significant role to prevent a surgery. Moreover, the percentage of men with lower urinary tract symptoms over age of 40 is slightly rising and counts $60 \%$ [7]. As so, the voiding dysfunction should have major impact on the life quality for a large proportion of men. The diagnosis is often based on urinary flow measurements evaluation, called uroflowmetry. The uroflowmetry (UF) is widely used non-invasive test, which evaluates emptying of the bladder [4].

The UF is basically carried out on an outpatient basis, at specific process involving the person urinate into the measurement device (uroflowmeter) at predetermined time. This procedure is not comfortable for the most patients. Fore-more, the voiding "on-demand" is unnatural, which causes the significant test-to-test variability [12]. As so, the test repetition is recommended, which is time-consuming and costly ineffective [11], [17]. Therefore, the demand for new small devices for comfortable use, but also for easy and practical methods signaling emerging problems, is growing. 
The one of mostly used urodynamic measurement method in clinical environment rests on weighing of the voided urine in time [19]. Since the dominant methods for plausible diagnoses are based on the urine flow, the derivative of weighted urine in time must be determined or calculated. Block diagram of the typical uroflowmeter widely used in clinical environment is shown in Fig. 1.

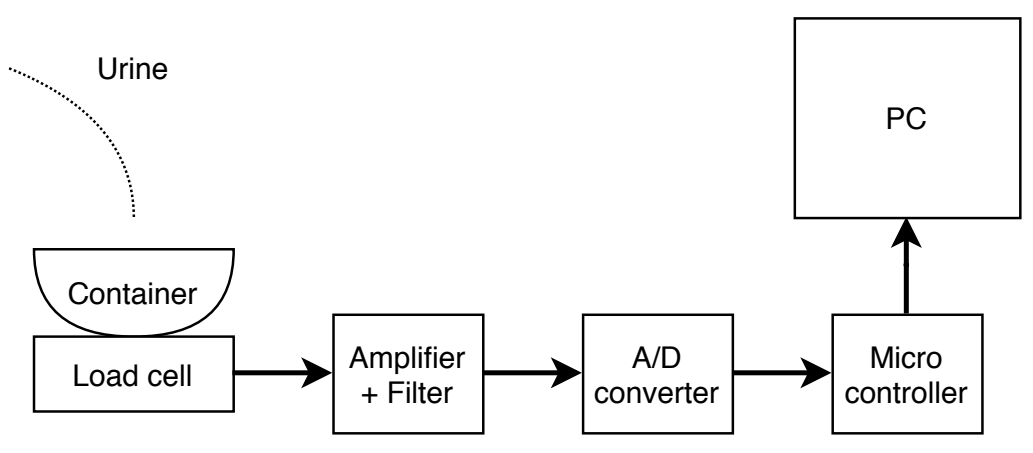

Fig. 1. Block diagram of uroflowmeter, where A/D converter stands for analog to digital converter and $\mathrm{PC}$ is personal computer.

When the urinary flow curves are obtained, the significant parameters are measured according to the recommendations of the ICS - International Continence Society [18]. Not only parameters are tracked, but it's also highly recommended to evaluate the whole urinary flow curve. At first, the curves can be divided into continuous and discontinuous types. For more, the continuous curves are divided into normal, box and compressive type [6]. These clusters often indicate a disease. However, since the curve evaluation is mostly performed by urologist or other specialist, the result may be affected by human error. Therefore, the obvious direction of the research is to develop and to propose a method capable of helping the doctor make decisions. Here, the authors try to propose a set of possible machine learning approaches in order to provide the mentioned capability.

In this article, the curves of the urinary flow and the urine weight in time, acquired by mostly used clinical uroflowmeter [19], are observed.

The article is structured as follows. At first the problem is formulated. This includes the description and examples of the typical UF curve shapes and parameters. In the next section, the proposed methods are introduced. Then the possible use is discussed. The article is finished with conclusions. 


\section{Problem formulation}

The lower urinary tract symptoms are diagnosed with urodynamic methods by default. Urodynamics is a study, where bladder and urethra are on the scope of view when performing their function of storing and releasing urine. One of mostly used method is an uroflowmetry. The UF is widely used, because of its benefits like non-invasiveness, easy reproduction, simple use, and possibility of repetition over time [3]. The aim of this paper is to propose and discuss a chain of steps necessary for the UF autonomous application.

The UF is widely researched topic based on analysis of bladder emptying divided into a two main branches. The first one is the analysis of the urinal flow curves, where mainly the shapes are observed. The second direction is about analysis of quantitative parameters. Casually, the measured data must be preprocessed for further use in mentioned directions.

\subsection{Curves selection}

The uroflowmetric curves measured in typical case cannot be automatically used before preprocessing. It is caused by voiding in unnatural conditions, where the beginning of urination is often repeated, total voiding amount is below minimal limit and the psychical influence occurs when patient urinates in the presence of another person (nurse, doctor). Thus, the only data where total volume of urine is bigger than $150 \mathrm{ml}$ are selected for further consideration as recommended in [15]. Then, from the whole measurement process, only the part from real start of urinating and its finish are chosen. As the raw data are considerably affected by noise, the filtering is executed. The curve selection and preprocessing for possible automatic processing is implied in Fig. 2.
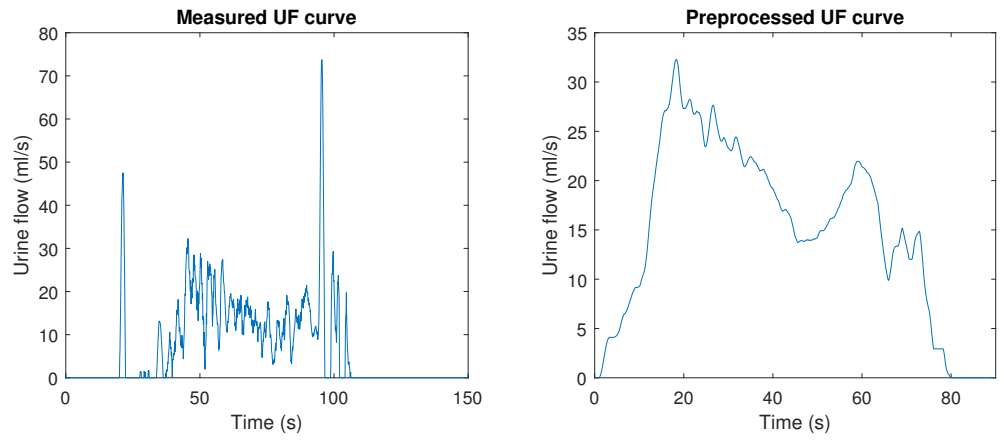

Fig. 2. Measured data from uroflowmeter and preprocessed data for possible use. 


\subsection{Curve types}

The basic diagnoses are based on the shape of the uroflowmetric curve. The uroflowmetric curve shows the flow of urine through the urethra captured in time. The standard uroflowmeters are measuring the total weight of urine voided into the container in defined time steps. This measurements must be processed to obtain the uroflowmetric curve. The curve shape for health patient is visibly different with patients with any lower urinary tract symptoms (LUTS).

The normal uroflometric curve, representing the patient with healthy lower urinary tract, have a typical bell shape.

Among the frequently observed LUTS, compressive and constrictive type can be mentioned, as was implied in Introduction section. The constrictive type arises with urethra stricture, where the curve have the box shape. The uroflowmetric curve of patient with enlarged prostate (BPH obstruction) has a special shape in between of bell and box shapes. The flow of urine in time graphs for normal and both mentioned obstructions are captured in Fig. 3 .
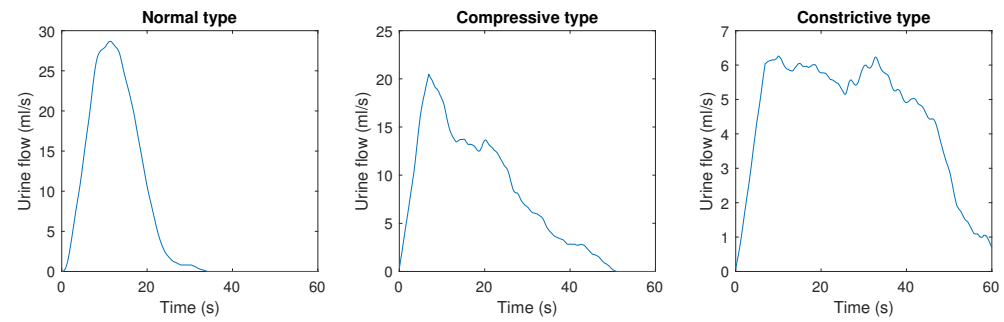

Fig. 3. Uroflowmetric curves.

\subsection{Quantitative parameters}

For a simplified information, only several parameters, summarizing main indicators of urine voiding process, can be used instead of the whole curve. The total volume of voided urine is the first parameter. As next, the maximal and average flow can be obtained as suitable parameters. As last major parameters, the urination time and time to reach maximum flow can be selected. These parameters are typically read from uroflowmetric curve by urologist, but could be also numerically calculated from data.

\section{Proposed methods}

As the types of curves are visually separated by urologist, the use of classification and clustering algorithms comes in consideration. Not only with the curves, but also with the quantitative parameters or other feature extraction technique, 
can be possibly the classification realized in practice. As the prevention plays a significant role, the main purpose should be just to separate the bell shape curves (healthy LUT) from others. Accordingly, the pattern recognition techniques should be applied.

\subsection{Pattern recognition system}

The process of recognizing patterns with machine learning methods is called pattern recognition. The pattern recognition algorithms are basically composed of sensing part, preprocessing part, feature extraction algorithm and description algorithm [14].

In pattern recognition system, the preprocessing is a process where data are divided into multiple segments based on obvious data difference. In the case of UF curves, the segmentation is based on LUT symptoms, which are changing curve shape.

The feature extraction mechanism starts on initial set of measured data and builds derived values intended to be informative and non-redundant. The different types of feature extraction applicable to UF data are described in the next section.

\subsection{Feature extraction}

As the UF curve is a typical graph containing a dependent variable changing in time, the different approaches of feature extraction could be realized. Fore-more, the urinal system should be described by the VBN model [20]. Here, individual feature extraction approaches are examined. For all possible approaches data must be preprocessed as described in section 2.1 and normalized.

Raw data as features This proposed approach is based on the idea that whole sequence of data could carry enough information for specific pattern recognition [16]. As so, the idea is about putting the normalized UF curve data to description algorithm with its output pairs represented by assigned class. The classes are sufficiently separable, which increases possibility of time series classification by this approach. The scheme is shown in Fig. 4.

Model parameters estimation From the physical essence, the weight of voided urine in time is some kind of transition characteristics. Thus, the general mathematical model of LUT can be defined. Model parameters should vary for each person. However, the correlation of parameters between patients with same symptoms could be detected.

One of methods is to try the data for model identification [2]. For this purpose the weight in time dependency data could be used. At first, the type and the complexity of the model should be defined. ARX, ARMAX, NARX, NARMAX or neural models are only a few of many possible architectures to be selected. 


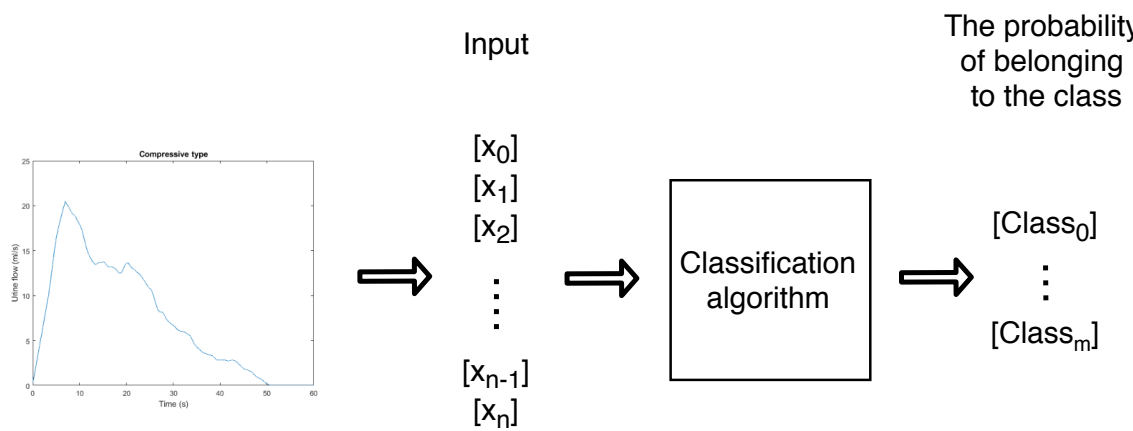

Fig. 4. Time series classification with raw data as features.

The mean square error between data and model can be also tested. Based on minimal error, the best model structure can be selected.

When the model structure is defined, the model parameters could be obtained by model training or fitting. As an input, a step function should be used with max value equal to total weight of voided urine and starting in time of voiding start. Based on model structure, the output could be affected by its previous values. Then, the model parameters should be used as an input to classification algorithm. An example of this approach, considering a neural model, is implied in Fig. 5.

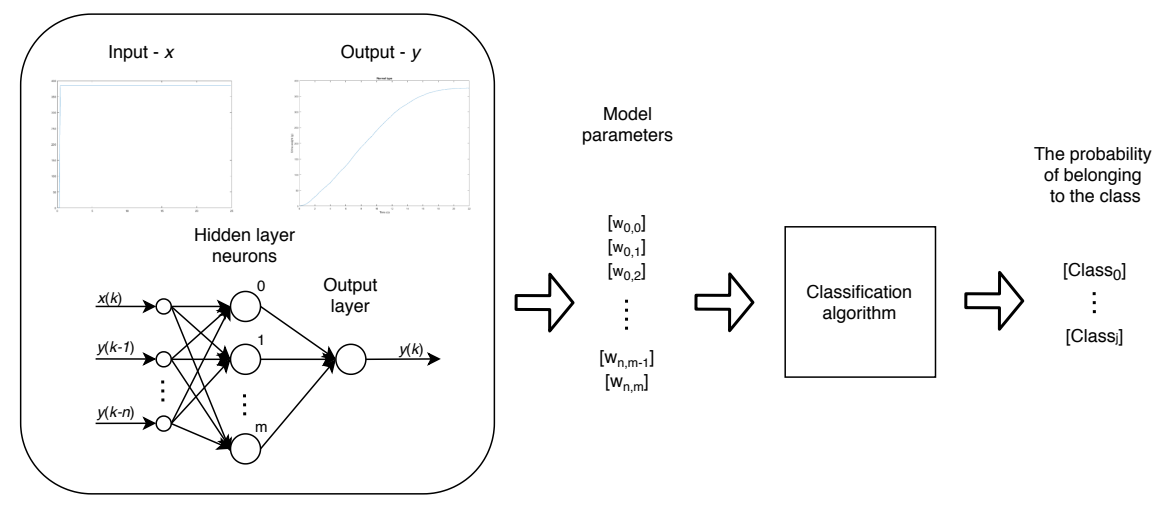

Fig. 5. Schema of the classification using parameters from trained neural model as an inputs. 
Polynomial approximation Based on same data, the characteristics can be interpolated with defined lines or should be approximated by splines or other mathematical functions. Finding the parameters is an optimization problem often solved by minimization of the mean square error. Subsequent to the data acquisition, the parameters of the splines or mathematical functions can be considered as an input for classification algorithm.

Quantitative parameters The quantitative parameters of UF curves are widely used as initial assessment of healthy patient in practice. One of the ideas is to test the possible classification with only quantitative parameters as an input data. Fig. 6.

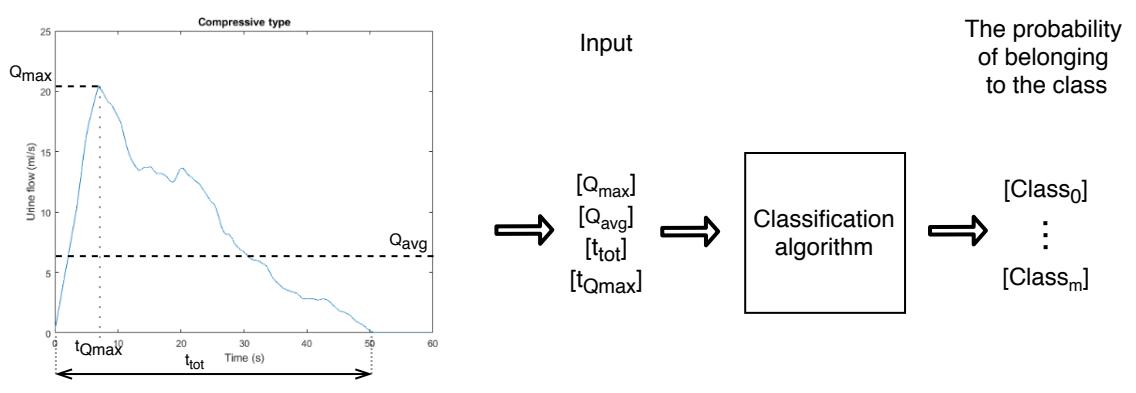

Fig. 6. Quantitative parameters used as features for classification.

\subsection{Description algorithm}

Providing a reasonable answer for all possible inputs is generally the main aim of pattern recognition systems. That can be basically achieved using classification methods, clustering or regression methods. As the classes are defined by urologist, the classification methods are on the scope of the article.

Classification trees The classification tree methods are used to predict class membership of a categorical dependent variable from their measurements on one or more predictor variables [13]. Predictor or ordered predictor variables categorical splits could be easily realized by binary trees. Not as only at binary decision, but also on linear combination splits the classification can be compute.

Nearest Neighbor In the pattern recognition, the k-nearest neighbor algorithms are used. It is a non-parametric method used for regression and classification. The input consist of the $k$ closest training examples in the feature space [1]. In the classification problem, the output is a class membership. An object is 
classified due to voting count of its neighbors, where it is assigned to the most common class among its $k$ nearest neighbors.

Naive Bayes The naive Bayes classification algorithms are based on the Bayes' theorem, which describes the probability of event, based on prior knowledge of conditions that might by related to the event [10]. Naive Bayes is a simple technique for constructing classifiers, which is the model that assign class labels to problem instances, represented as vectors of feature values.

SVM classification The main objective of the support vector machine algorithm is to find a hyperplane in a $N$-dimensional space, where the $N$ is the number of features, that clearly classifies the data points [5].

Feedforward Neural networks The main ability of the neural networks is that they can learn complex non-linear input-output relationships. That is achieved by the sequential training procedures and by great self adeptness to the data [9]. The most commonly used family of the neural networks for the classification task is the feedforward neural network [8].

The feedforward neural network (FFNN) is composed by the input layer, where every input variable must be connected to its neuron, then by the hidden layers and finally by the output layer, where the count of neurons in the output layer is the same as count of outputs. The learning process involves updating of neuron connections weights, which makes the FFNN capable to perform clustering and classification tasks.

\section{Conclusion}

In the article were presented methods and designs for an automatic uroflow curves recognition and classification. Urologists often use only several indicators on the intuitive bases for the urinary flow curve classification, which in hand with knowing of patient history could lead to clear LUTS diagnoses. According to this idea, the use of only classical recognition methods could finish with failure. As such, the main objective is to use a different approaches for features extraction. The probability of successful LUTS diagnose is increasing with the feature extraction methods diversity. The presented methodology and designs tries to keep the same idea and for that could provide the base for the automatic uroflow curves classification.

\section{Discussion and feature work}

As a lot of the clinical measurement devices uses weighting of the voided urine carried out on outpatient basis, the urinary flow curve can be considerably distorted. The data acquisition in natural environment should be preferred, even at 
the cost of reduced quality caused by unprofessional measurement. Hence, the automatic classification could help with LUT diagnoses based on knowledge of the patient LUT model.

For possible classification of lower urinary track symptoms a several approaches were introduced. In the further work, the methods will be reviewed on extensive dataset, which is currently being created. As the credible dataset verified by several urologist will be obtained, the proposed methods should be examined. Direction of further development will depend on the results of introduced methods. If the results of the methods are comparably accurate with the urologist decisions, their further extensions will be researched. In the other case, the new concepts for LUTS classification must arise.

\section{Acknowledgment}

The work has been supported by the IGA Funds of the University of Pardubice, Czech Republic. This support is very gratefully acknowledged.

\section{References}

1. Altman, N.S.: An introduction to kernel and nearest-neighbor nonparametric regression. The American Statistician 46(3), 175-185 (1992), https://www. tandfonline.com/doi/abs/10.1080/00031305.1992.10475879

2. Billings, S.A.: Nonlinear system identification: NARMAX methods in the time, frequency, and spatio-temporal domains. Chichester, West Sussex, United Kingdom: John Wiley \& Sons (2013)

3. Buresova, E., Vidlar, A., Student, V.: Uroflowmetrie, nenahraditelna vysetrovaci metoda $\mathrm{k}$ diagnostice mocovych dysfunkci. Urology for Practice 14(4), 170-172 (2013), https://www.urologiepropraxi.cz/artkey/uro-201304-0006.php

4. Chua, M.E., Mendoza, J., See, M., Esmena, E., Aguila, D., Silangcruz, J.M., Reyes, B.J., Luna, Jr., S., Morales, Jr., M.: A critical review of recent clinical practice guidelines on the diagnosis and treatment of non-neurogenic male lower urinary tract symptoms. CUAJ-CANADIAN UROLOGICAL ASSOCIATION JOURNAL 9(7-8), E463-E470 (JUL 2015)

5. Cortes, C., Vapnik, V.: Support-vector networks. Machine Learning 20(3), 273-297 (Sep 1995), https://doi.org/10.1007/BF00994018

6. Drake, M.J., Doumouchtsis, S.K., Hashim, H., Gammie, A.: Fundamentals of urodynamic practice, based on International Continence Society good urodynamic practices recommendations. NEUROUROLOGY AND URODYNAMICS 37(6), S50-S60 (AUG 2018)

7. Irwin, D.E., Milsom, I., Hunskaar, S., Reilly, K., Kopp, Z., Herschorn, S., Coyne, K., Kelleher, C., Hampel, C., Artibani, W., Abrams, P.: Population-based survey of urinary incontinence, overactive bladder, and other lower urinary tract symptoms in five countries: Results of the EPIC study. EUROPEAN UROLOGY 50(6), 13061315 (DEC 2006)

8. Jain, A.K., Jianchang Mao, Mohiuddin, K.M.: Artificial neural networks: a tutorial. Computer 29(3), 31-44 (March 1996) 
9. Jayanta, Bhattacharyya, D., Kim, T.H.: Use of artificial neural network in pattern recognition. International Journal of Software Engineering and Its Applications 4, 23-33 (05 2010)

10. Josang, A.: Generalising Bayes' Theorem in Subjective Logic. In: 2016 IEEE INTERNATIONAL CONFERENCE ON MULTISENSOR FUSION AND INTEGRATION FOR INTELLIGENT SYSTEMS (MFI). pp. 462-469. IEEE (2016), IEEE International Conference on Multisensor Fusion and Integration for Intelligent Systems (MFI), Baden-Baden, GERMANY, SEP 19-21, 2016

11. Kranse, R., van Mastrigt, R.: Causes for variability in repeated pressure-flow measurements. UROLOGY 61(5), 930-934 (MAY 2003)

12. Krhut, J., Gaertner, M., Sykora, R., Hurtik, P., Burda, M., Lunacek, L., Zvarova, K., Zvara, P.: Comparison between uroflowmetry and sonouroflowmetry in recording of urinary flow in healthy men. INTERNATIONAL JOURNAL OF UROLOGY 22(8), 761-765 (AUG 2015)

13. Lahoti, S., Mathew, K., Miner, G.: Tutorial h - predictive process control: Qcdata mining using statistica data miner and qc-miner. In: Nisbet, R., Elder, J., Miner, G. (eds.) Handbook of Statistical Analysis and Data Mining Applications, pp. 513 - 530. Academic Press, Boston (2009), http://www.sciencedirect.com/ science/article/pii/B9780123747655000309

14. Little, M.A.: Machine learning for signal processing: data science, algorithms, and computational statistics. New York: Oxford University Press (2019)

15. Madersbacher, S., Alivizatos, G., Nordling, J., Sanz, C., Emberton, M., de la Rosette, J.: EAU 2004 guidelines on assessment, therapy and follow-up of men with lower urinary tract symptoms suggestive of benign prostatic obstruction (BPH guidelines). EUROPEAN UROLOGY 46(5), 547-554 (NOV 2004)

16. Pan, L., Meng, Q., Pan, W., Zhao, Y., Gao, H.: A Feature Segment Based Time Series Classification Algorithm. In: Li, JB (ed.) 2015 FIFTH INTERNATIONAL CONFERENCE ON INSTRUMENTATION AND MEASUREMENT, COMPUTER, COMMUNICATION AND CONTROL (IMCCC). pp. 1333-1338. International Conference on Instrumentation Measurement Computer Communication and Control, Harbin Inst Tech; Yanshan Univ; NE Univ Qinhuangdao; IEEE Instrumentation and Measurement Soc; IEEE IM Soc, Beijing \& Harbin Joint Chapter; Heilongjiang Instrument and Measurement Soc; IEEE Computer soc (2015), Fifth International Conference on Instrumentation \& Measurement, Computer, Communication, and Control (IMCCC), Qinhuangdao, PEOPLES R CHINA, SEP 18-20, 2015

17. de la Rosette, J., Witjes, W., Schafer, W., Abrams, P., Donovan, J., Peters, T., Millard, R., Frimodt-Moller, C., Kalomiris, P., Grp, I.B.S.: Relationships between lower urinary tract symptoms and bladder outlet obstruction: Results from the ICS-"BPH" study. NEUROUROLOGY AND URODYNAMICS 17(2), 99-108 (1998)

18. Schafer, W., Abrams, P., Liao, L., Mattiasson, A., Pesce, F., Spangberg, A., Sterling, A., Zinner, N., van Kerrebroeck, P.: Good urodynamic practices: Uroflowmetry, filling cystometry, and pressure-flow studies. NEUROUROLOGY AND URODYNAMICS 21(3), 261-274 (2002)

19. Urbonavicius, B.G., Kaskonas, P.: Urodynamic measurement techniques: A review. MEASUREMENT 90, 64-73 (AUG 2016)

20. Valentini, F.A., Besson, G.R., Nelson, P.P., Zimmern, P.E.: Clinically relevant modeling of urodynamics function: The VBN model. NEUROUROLOGY AND URODYNAMICS 33(3), 361-366 (MAR 2014) 